\title{
25 años de la CONAMED en números
}

\author{
25 years of CONAMED in numbers
}

Onofre Muñoz Hernández*

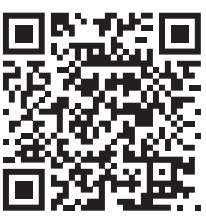

Quiero hacer énfasis en lo mencionado en la introducción en relación con la misión y destacar que la función más clara de la Comisión Nacional de Arbitraje Médico (CONAMED) es la avenencia entre los usuarios y los prestadores de servicios médicos, pero también la colaboración con las autoridades de procuración de justicia en la elaboración de dictámenes médicos, la generación de investigación sobre el quehacer institucional, y la emisión de recomendaciones y acciones educativas para contribuir a mejorar la calidad de la atención médica y la seguridad del paciente.

Los objetivos derivan de la misión: el primero se refiere a impulsar la solución de quejas derivadas del uso de los servicios de salud a través de las acciones de orientación, gestión, conciliación y arbitraje; el segundo se relaciona con analizar las causas y consecuencias de los incidentes en salud, haciendo revisiones periódicas de la experiencia que tenemos y tercero, fortalecer la gestión institucional a través de la mejora continua.

Analizamos los 25 años de experiencia en forma de quinquenios y utilizamos la mediana para un mejor manejo de la dispersión de los datos; son medianas por cada cinco años de trabajo. En cuanto a los asuntos concluidos, nos hemos mantenido al nivel de 15,000 por año (Figura 7); las orientaciones también se han mantenido alrededor de 10,000 (Figura 2). En cuanto a asesorías especializadas, éstas varían de 3,000 a 4,000 en los últimos 10 años (Figura 3); lo que sí ha aumentado de manera clara son las gestiones inmediatas (Figura 4), ya que la población ha identificado que es un mecanismo que les permite resolver rápidamente alguna inconformidad con la relación médico-paciente inicial o con la institución de salud como problemas de citas en consultas, el diferimiento de servicios o requerimientos adicionales y la respuesta de las instituciones de salud ha sido muy favorable en relación a la solicitud de un paciente cuando su expectativa es recibir una atención oportuna.

En relación a los principales motivos de queja, siempre han estado el tratamiento médico, el tratamiento quirúrgico y el diagnóstico, pero en los últimos 10 años se han agregado problemas de la relación médico-paciente, que es un componente importante de la calidad de la atención y que el usuario identifica como deficiente o problemática (Figura 5).

En cuanto a los servicios de especialidad involucrados en las quejas, también se repiten en el tiempo la cirugía general, traumatología y ortopedia, ginecología y obstetricia y los servicios de urgencias; a través del tiempo las quejas sobre odontología y sus especialidades han ocupado ya un lugar importante en los últimos 10 años y actualmente son el primer motivo de causa de inconformidad a nivel nacional (Figura 6).
* Comisionado Nacional de Arbitraje Médico.

\section{Correspondencia:}

OMH,omunoz@ conamed.gob.mx Conflicto de intereses: Sin conflicto de intereses. Citar como: Muñoz HO. 25 años de la CONAMED en números. Rev CONAMED. 2021; 26(4): 173-178. https:// dx.doi.org/10.35366/102504 Financiamiento: No hubo financiamiento.

Recibido: 15/10/2021. Aceptado: 15/10/2021. 


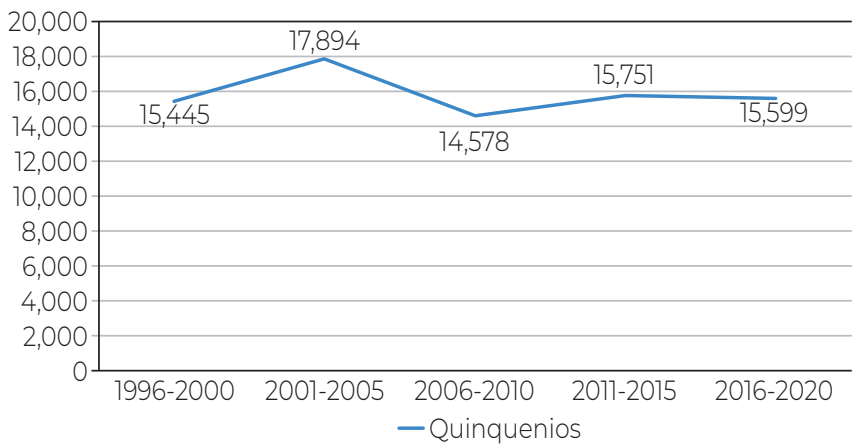

Figura 1: Total de asuntos concluidos 1996-2020 (mediana quinquenal).

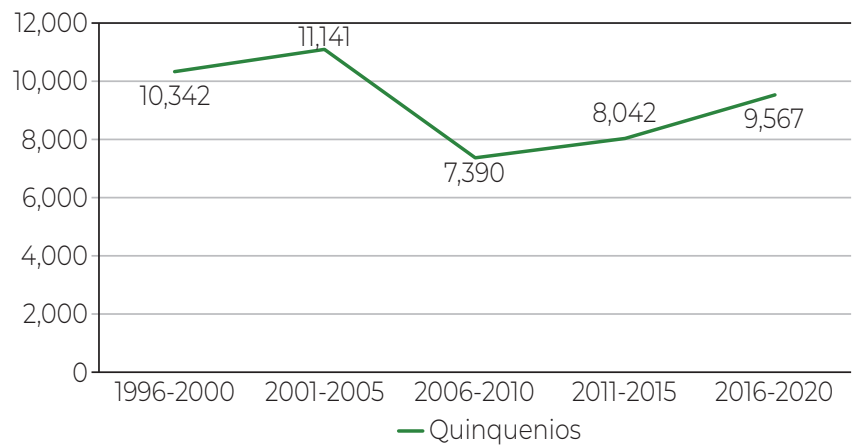

Figura 2: Total de orientaciones 1996-2020 (mediana quinquenal).
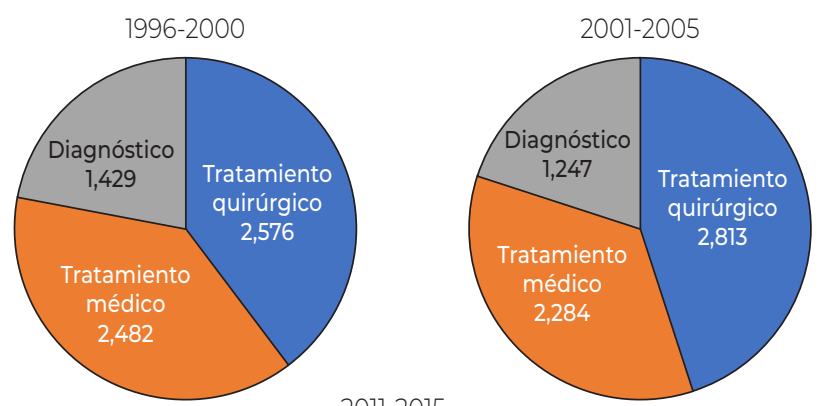

$2011-2015$

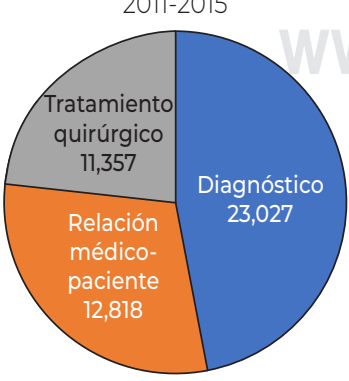

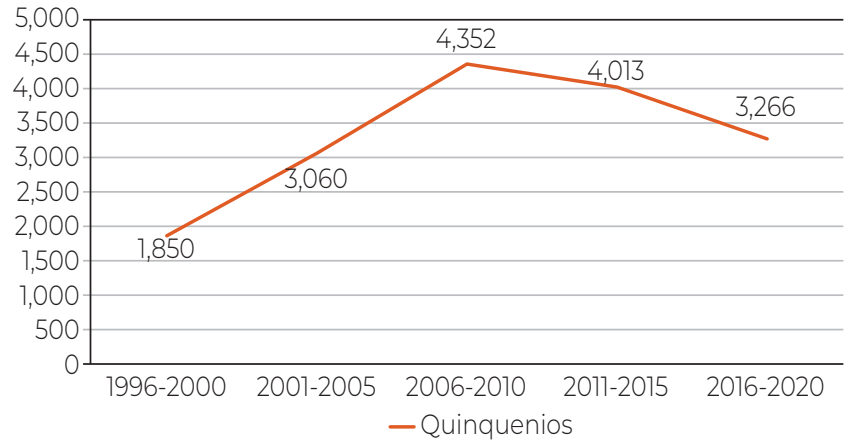

Figura 3: Total de asesorías especializadas 1996-2020 (mediana quinquenal).

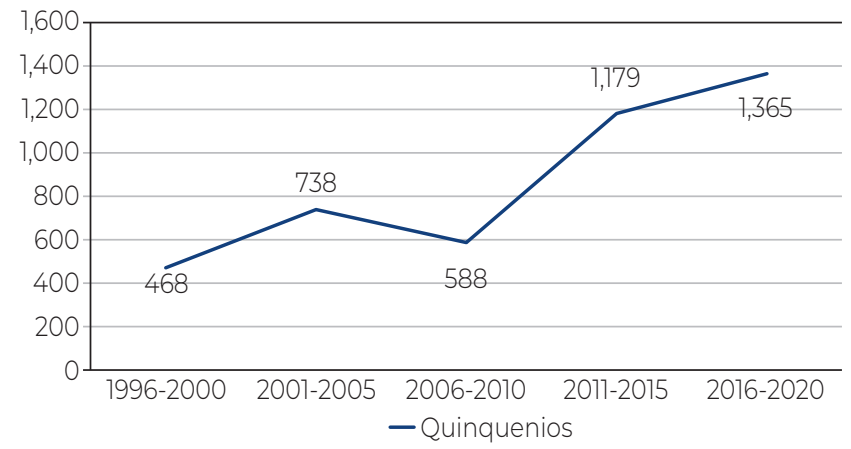

Figura 4: Total de gestiones inmediatas 1996-2020 (mediana quinquenal).

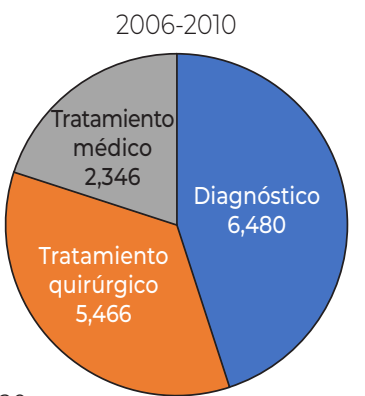

2016-2020

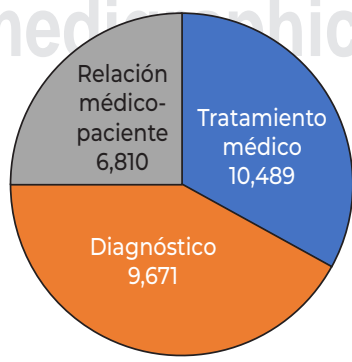

Figura 5:

Tres principales motivos mencionados por los usuarios en las quejas concluidas según quinquenio 2016-2020. 
Figura 6:

Cinco principales servicios médicos involucrados en las quejas concluidas según quinquenio 1996-2020.
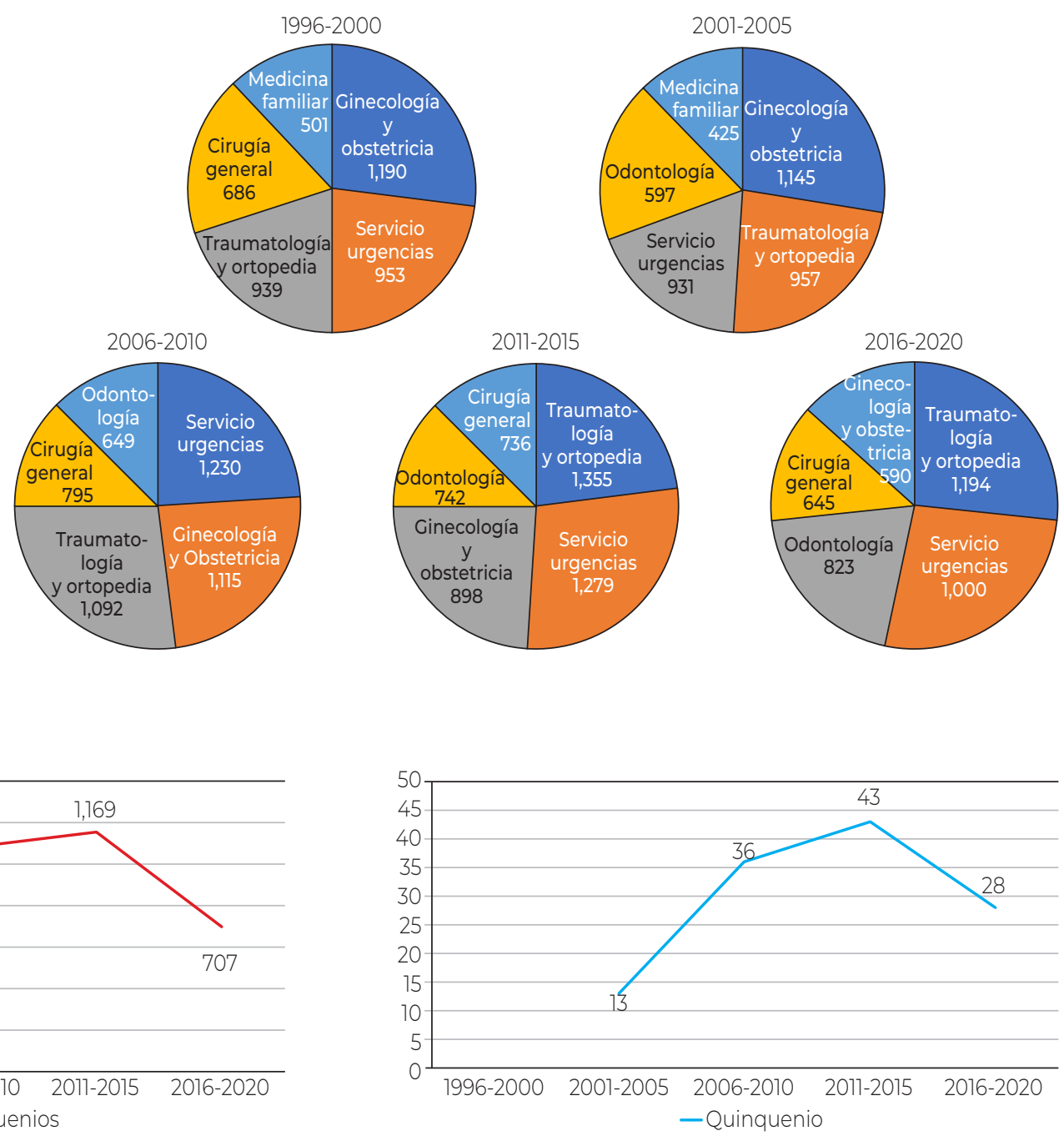

Figura 9: Total de laudos condenatorios 1996-2020 (mediana quinquenal).

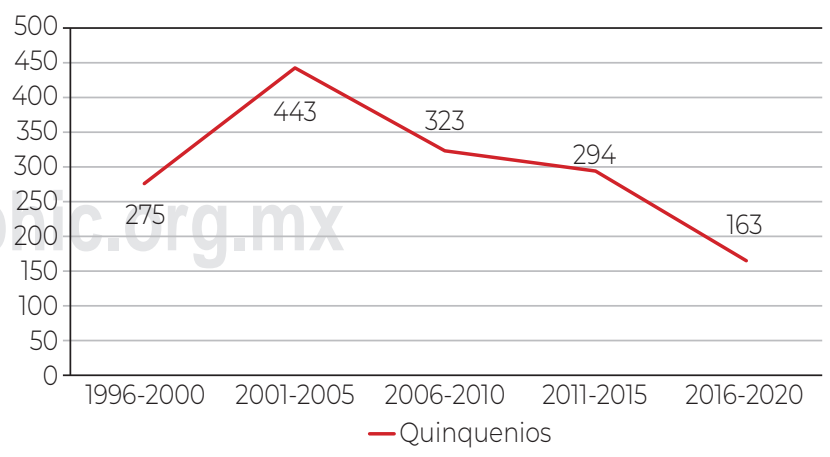

Figura 10: Total de dictámenes médicos institucionales concluidos 1996-2020 (mediana quinquenal).
Figura 8: Total de laudos emitidos 1996-2020 (mediana quinquenal).
Figura 7: Total de quejas concluidas por conciliación 19962020 (mediana quinquenal).

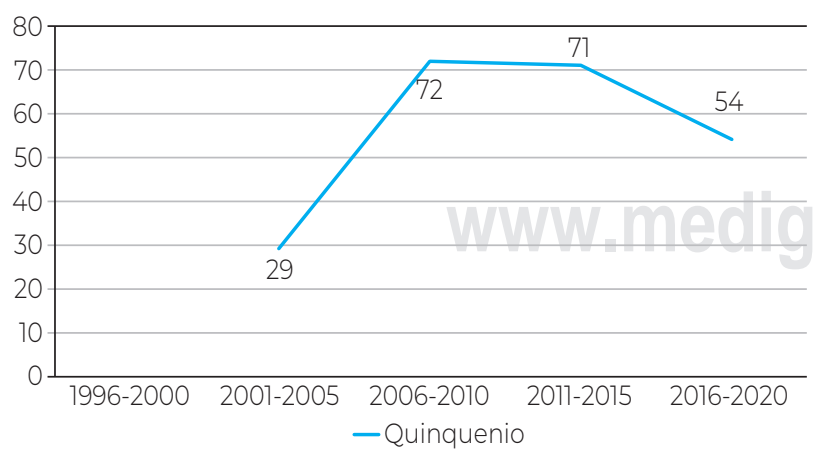


En relación a las quejas que se resuelven por conciliación, que son aproximadamente $40 \%$, se han mantenido en el orden de 1,000 anuales con variaciones (Figura 7); sólo $5 \%$ de los casos de quejas llegan al arbitraje y se resuelven por laudos entre 50-70 por año (Figura 8). Los médicos están muy atentos a la proporción de laudos condenatorios, la cual es de cerca de 50\%; tuvieron un ascenso muy importante en

Tabla 1: Cronología de la creación de las comisiones estatales de arbitraje médico y su naturaleza jurídica.

\begin{tabular}{cc}
\hline Estado (año de fundación) & Naturaleza jurídica \\
\hline Tabasco (1997) & Descentralizado \\
Estado de México (1998) & Descentralizado \\
Guanajuato (1998) & Descentralizado \\
Colima (1998) & Desconcentrado \\
San Luis Potosí (1998) & Desconcentrado \\
Veracruz (1999) & Desconcentrado \\
Querétaro (1999) & Desconcentrado \\
Aguascalientes (1999) & Descentralizado \\
Guerrero (1999) & Desconcentrado \\
Puebla (1999) & Desconcentrado \\
Michoacán (2000) & Descentralizado \\
Campeche (2000) & Desconcentrado \\
Morelos (2000) & Descentralizado \\
Tamaulipas (2000) & Desconcentrado \\
Sinaloa (2000) & Desconcentrado \\
Nayarit (2000) & Descentralizado \\
Jalisco (2001) & Descentralizado \\
Chiapas (2001) & Descentralizado \\
Baja California (2001) & Descentralizado \\
Tlaxcala (2002) & Descentralizado \\
Nuevo León (2002) & Desconcentrado \\
Yucatán (2003) & Desconcentrado \\
Coahuila (2004) & Autónoma \\
Oaxaca (2004) & Desconcena \\
Sonora (2007) & Desconcentrado \\
Hidalgo (2011) & Desconcentrado \\
Chihuahua (2018) & Descentralizado \\
Baja California Sur (2018) & \\
\hline & \\
\hline
\end{tabular}

los primeros 15 años y después han empezado a descender (Figura 9). Lo que sí es claro, es el descenso de las solicitudes de dictámenes médicos institucionales por las instituciones de impartición de justicia, donde participamos como terceros (Figura 10).

En el año 2002 se crea por decisión de todas las comisiones estatales y la CONAMED el Consejo Mexicano de Arbitraje Médico (CMAM); tenemos un convenio de coordinación firmado por todos, y con el secretario de salud como testigo de honor. Ya somos 28 comisiones (Tabla 7), como ven la primera en Tabasco en 1997 y la última en Baja California Sur en 2018, todavía faltan tres: Durango, Zacatecas y Quintana Roo, que ya cuentan con su instrumento jurídico de creación, pero aún no cuentan con las instalaciones para lograr la estructura y poder iniciar sus actividades.

En 2016 decidimos en el CMAM elaborar y diseñar una plataforma de registro para poder analizar los resultados de todos los estados y tener una información nacional global que es muy necesaria porque individualmente no tenemos suficiente material para hacer ciertos análisis poblacionales. La Tabla 2 muestra que anualmente atendemos 40,000 asuntos, excepto en 2020 que fue un año atípico por la pandemia y es claro que todas las comisiones tuvimos un decrecimiento en nuestras actividades.

Como decía, necesitamos contar con un volumen grande de información para poder empezar a utilizar denominadores poblacionales, y tener mejor información sobre qué está ocurriendo en el país. Voy a presentar sólo dos ejemplos: el denominador es 10,000 egresos hospitalarios (quejas por egreso hospitalario) y se puede ver, sin tomar en cuenta el año 2020 que es un año atípico, en color verde la tasa para el sector público que varía entre tres a cuatro por cada 10,000 egresos versus la del sector privado; y en color rojo, que varía entre siete y ocho quejas por cada 10,000 egresos hospitalarios y que se ha mantenido en los últimos años (Figura 77).

En esta Figura 12 podemos ver los laudos emitidos en los sectores público y privado. Tenemos una variación interesante: Ios primeros dos años 60\%, en color verde, eran absolutorios y $40 \%$ condenatorios, en 2018 se equiparan 50\% absolutorios y 50\% condenatorios, y en 2019-2020 se invirtió 60\%, que 
Tabla 2: Principales asuntos concluidos por las comisiones estatales de Arbitraje Médico, análisis comparativo 2016-2020.

\begin{tabular}{lrrrrrr}
\hline & \multicolumn{5}{c}{ Asuntos concluidos } \\
\cline { 2 - 5 } Tipo de asunto & 2016 & 2017 & 2018 & 2019 & 2020 & \% variación \\
\hline Orientaciones & 22,066 & 22,975 & 18,850 & 22,961 & 13,214 & -42.5 \\
Asesorías especializadas & 8,921 & 8,769 & 8,874 & 10,381 & 8,379 & -19.3 \\
Gestiones inmediatas & 5,066 & 5,767 & 4,425 & 5,194 & 3,932 & -24.3 \\
Quejas & 3,757 & 3,801 & 3,060 & 2,812 & 1,687 & -40.0 \\
Dictámenes & 611 & 699 & 588 & 468 & 369 & -21.2 \\
Opinión técnico-médica & 127 & 162 & 169 & 125 & 98 & -21.6 \\
Total & 40,548 & 42,173 & 35,966 & 41,941 & 27,679 & -34.0 \\
\hline
\end{tabular}

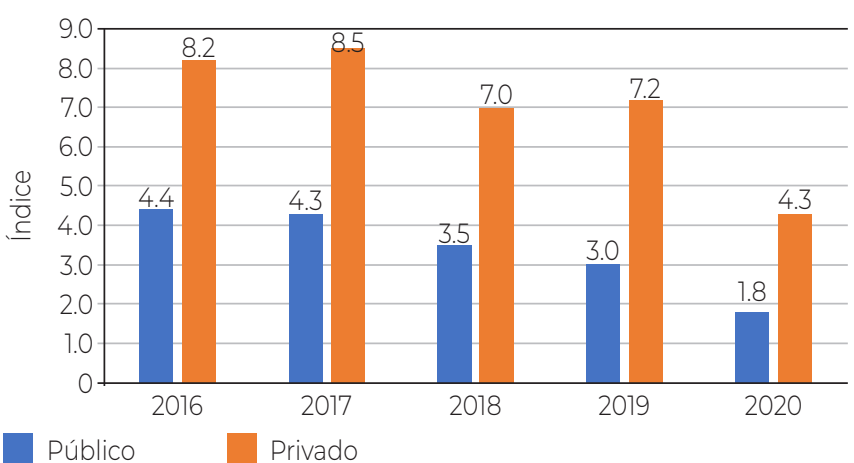

Figura 11: Índice de quejas por cada 10 mil egresos según sector involucrado (público, privado), análisis comparativo 2016-2020.

Fuente: plataforma de registro de quejas médicas y dictámenes.

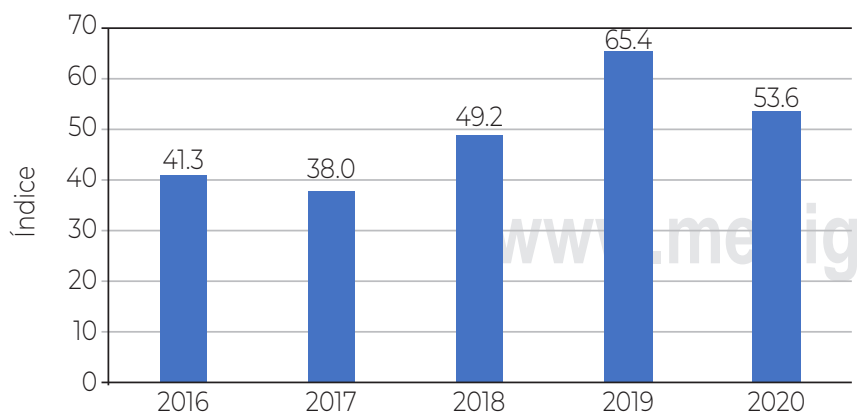

Condenatorio

Figura 12: Laudos concluidos según sentido de resolución, análisis comparativo 2016-2020. son condenatorios y $40 \%$ absolutorios. El tiempo confirmará la tendencia.

Cumple 25 años nuestro órgano oficial de difusión que es la Revista CONAMED que también nace en 1996; las autoridades que decidieron la creación de la CONAMED, y retomo otra vez el agradecimiento en especial al Dr. Juan Ramón de la Fuente y al Dr. Héctor Fernández Varela quien fue el operador, como ya escuchamos, para que se hiciera realidad lo que estaba plasmado en el papel. El primer número salió en 1996 y en 2021 tenemos ya el número 1 del año 2021 (Figura 13). Ha habido continuidad en nuestro órgano de difusión, es una publicación trimestral que está registrada en los índices fundamentalmente latinoamericanos, se publican cuatro números por año y un suplemento, que a partir de 2018 existe sólo en formato electrónico.

El consejo editorial está constituido por una mezcla de organizaciones internacionales o extranjeras con organizaciones nacionales. Es una revista muy visitada, tenemos un promedio de consultas diarias de cerca de 2,000, quizá lo más importante son los artículos de descarga completa, con 1,131 descargas diarias. Es una revista muy consultada por los temas que trata sobre la calidad de la atención y la seguridad del paciente y de educación médica continua en salud, poco citada, tiene citas muy bajas para los estándares internacionales, pero creo que el lugar que 


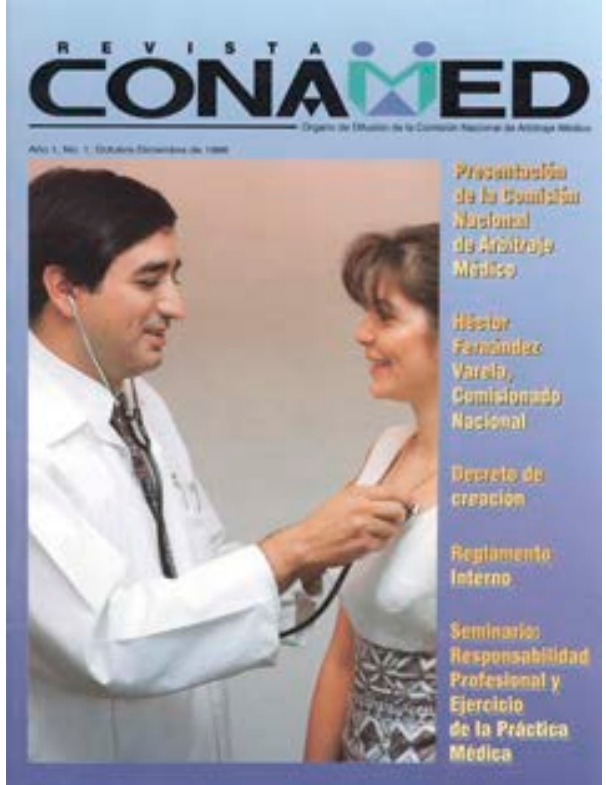

Vol. 1, Núm. 1, 1996

\section{REVISTA CONAB̈ED}
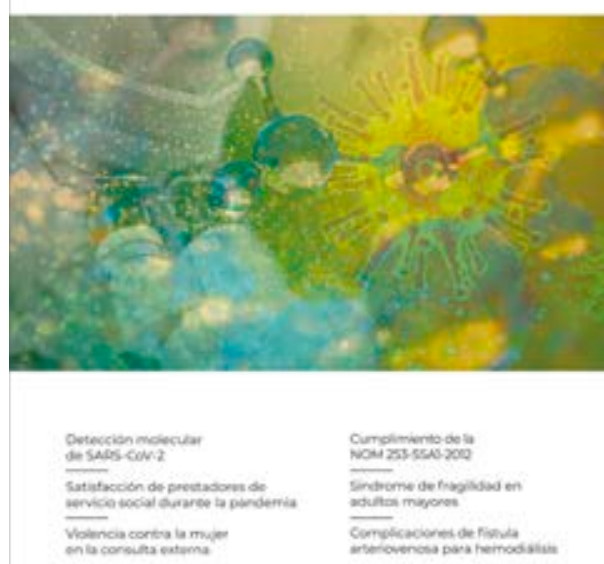

Vol. 26, Núm. 1, 2021
Figura 13:

Revista Comisión Nacional de Arbitraje Médico (CONAMED). tenemos está en el sentido de las consultas que se hacen a nuestra revista sobre los temas que tratamos o que divulgamos y porque es nuestro órgano oficial de difusión hacia la comunidad de salud nacional e internacional.

Traté de forma breve de dar una imagen de los primeros 25 años de trabajo de la CONAMED. Existe una inquietud en lo que significa ser más productivo en un organismo como la CONAMED: tener más números, incrementar al infinito las quejas o mantenerse en un nivel que permita su solución y que no crezcan; de hecho las tres primeras acciones que realizamos como asesoría especializada, gestión inmediata y consultoría son preventivas, tratando de evitar que el problema lleve a generar una queja y seremos más exitosos en el tiempo con un equilibrio que parece empezar a verse, donde las gestiones y las asesorías especializadas disminuyan el número de quejas y las instituciones de salud resuelvan mejor los problemas. El tiempo nos irá diciendo si al mejorar la calidad de la atención, las quejas disminuyen por 1,000 egresos o por 1,000 consultas o por 1,000 procedimientos quirúrgicos o si la seguridad del paciente se incrementa o si la certificación, que no es competencia nuestra, toma en cuenta las conciliaciones y los términos de laudos condenatorios o absolutorios para la calificación del hospital. Tenemos que ir conjuntando indicadores con otros organismos para medir el impacto en lo que queremos de la calidad de la prestación de servicios médicos en nuestras instituciones de salud.

\section{www. medig raphic. org.mx}

